J. Clin. Chem. Clin. Biochem.

Vol. 18, 1980, pp. 937-945

\title{
Analytik und biologische Bedeutung der Steroidepoxide ${ }^{1}$ )
}

\author{
Von H. Breuer
}

Institut für Klinische Biochemie der Universität Bonn

Sehr geehrter Herr Präsident, sehr verehrter, lieber Herr Staudinger, meine sehr geehrten Damen und Herren,

es ist für mich eine große Ehre und Freude zugleich, anläßlich der Verleihung der Scherer-Medaille an Sie, sehr verehrter Herr Staudinger, den Festvortrag halten zu dürfen. Eine Freude ist es besonders deshalb, weil ich auf diese Weise ein wenig von dem Respekt, den ich Ihrem Werk und Ihrer Persönlichkeit zolle, zum Ausdruck bringen kann. Unsere Wege haben sich immer wieder gekreuzt - zuerst bei den Mosbacher Colloquien der Fünfziger Jahre, als Sie für uns Jüngere der Promotor der Steroidanalytik in Deutschland waren. Viele Arbeiten sind damals durch Ihre Untersuchungen über die Nebennierenrinden-Hormone angeregt worden.

Es folgte die Zeit, in der man sich hauptsächlich auf Tagungen und Kongressen - leider oft nur allzu flüchtig - sah, bis wir uns dann bei der Deutschen Forschungsgemeinschaft in zunehmend intensiver Weise trafen. Sie waren Vizepräsident für die Biowissenschaften, und zwar - wie ich bald selber bemerkte und wie auch von allen Ihren Kollegen ohne Einschränkung anerkannt wurde - ein hochengagierter, kritisch abwägender und besonders den Problemen der Nachwuchsförderung aufgeschlossener Vizepräsident.

Ihre Tätigkeit bei der Deutschen Forschungsgemeinschaft hat in den Jahren der raschen Ausdehnung und der Ubernahme neuer Aufgaben entscheidend dazu beigetragen, daß die Entwicklung in die richtigen Bahnen gelenkt wurde und insbesondere politische Einflüsse auf die Forschung und die Forschungsförderung mit Erfolg abgewehrt werden konnten. Sie haben als Vizepräsident der Deutschen Forschungsgemeinschaft - und darüberhinaus in vielen anderen, ebenfalls wịchtigen Funktionen - viele Vorträge gehalten und Zeichen gesetzt, die einen großen persönlichen Mut erforderten. Dafür gebührt Ihnen der Dank der Gelehrtenrepublik.

Ihr Interesse an der Forschung ist nicht erlähmt, und das gibt mir den Mut, heute über eigene Arbeiten zu sprechen, die in einem gewissen Zusammenhang mit Ihrem wissenschaftlichen Werk auf dem Gebiet der Steroidhormone stehen. Es handelt sich um Untersuchungen über Steroidepoxide und die damit verbundenen Probleme der Steroidanalytik. Aus methodischen Gründen zogen sịch diese Studien über viele Jahre hin und konnten erst jetzt zu einem vorläufigen Abschlu $\vec{B}$ gebracht werden.

1) Als Festvortrag gehalten am 4. Juni 1980 in Freiburg/Brsg. anläßlich der Verleihung der Scherer-Medaille an Professor Dr. Hj. Staudinger. 
Ende der Fünfziger Jahre wurde bekannt, daß Ring D-ungesättigte Steroide, die bereits früher beim Eber (1) gefunden worden waren, auch beim Menschen vorkom$\operatorname{men}(2,3,4)$.

Dabei handelte es sich um $\Delta^{16}$-Steroide der Androgenreihe, die - zunächst hypothetisch - aus Testosteron durch Wasserabspaltung entstanden sein konnten und als Ring A-reduzierte Verbindungen im Urin (5) nachgewiesen wurden.

Die Umwandlung des $\Delta^{16}$-Steroids der Androgenreihe in das entsprechende Steroid der Östrogenreihe war mit Hilfe des aromatisierenden Enzymsystems der menschlichen Placenta möglich (6).

Da wir uns damals vorzugsweise mit Östrogenen befaß̣ten, interessierten wir uns für das Schicksal des phenolischen $\Delta^{16}$-Steroids.

Es zeigte sich, daß Rattenleberschnitte Östratetraen-3-ol rasch zu einem Isomeren von Östriol - und zwar zum $16 \beta, 17 \alpha$-Epiöstriol - umwandelten (7).

Wir postulierten als Zwischenprodukt ein $16 \alpha, 17 \alpha$ Epoxid und glaubten zunächst auch, mit Hilfe der Papierchromatographie die Bildung dieses Epoxides nachgewiesen zu haben.

Um diese Befunde auf eine breitere Basis zu stellen, wandten wir uns dem Stoffwechsel von Östratetraen-3-ol beim Menschen zu.

Tabelle 1 zeigt eine Zusammenstellung derjenigen Reaktionen, die vor etwa 20 Jahren zur Identifizierung von Steroiden herangezogen wurden.

Auch beim Menschen konnte 16 $\alpha, 17 \alpha$-Epiöstriol als Metabolit von Östratetraenol nachgewiesen werden (8). In weiteren Versuchen prüften wir nun das Verhalten der beiden sterisch möglichen Epoxide beim Menschen und fanden als Metaboliten des $16 \alpha, 17 \alpha$-Epoxids ausschließlich 16,17-Epiöstriol (das auch aus Östratetraen-3-ol entstand), während das $16 \beta, 17 \beta$-Epoxid sowohl 16,17-Epiöstriol als auch Östriol lieferte (8).

Diese Ergebnisse veranlaßten uns, einen SteroidepoxidStoffwechselweg auch beim Menschen anzunehmen, wobei allerdings eine Einschränkung gemacht werden mußte: Weder in vivo noch in vitro konnten wir die Epoxid-Verbindung selber mit Sicherheit nachweisen.
Offenbar wurde das gebildete Epoxid sehr rasch umgesetż, so daß die Zwischenstoffkonżentrationen außerordentlich gering und infolgedessen mit den damaligen steroidanalytischen Methoden nicht erfaßbar waren. Wir konnten also dieses Problem nicht weiter verfolgen und mußten die endgültige Klärung auf einen späteren Zeitpunkt vertagen.

Tab. 1. Zusammenstellung der Reaktionen zur Isolierung und Identifizierung von 16,17-Epiöstriol aus Urin nach Injektion von Östratetraenol, zitiert nach $1 . c$. (8).

\begin{tabular}{|c|c|c|}
\hline Untersucht & Metabolit & $\begin{array}{l}\text { Authen- } \\
\text { tisches } \\
16,17-\text {-Epi- } \\
\text { östriol }\end{array}$ \\
\hline
\end{tabular}

Wanderungsgeschwindigkeit auf Formamid-imprägniertem Papier im System Chloroform-Ethylacetat $(5+1)$ in $\mathrm{cm} / \mathrm{h} \quad 1,23 \quad 1,25$

Fp. nach Mikrosublimation $\left[{ }^{\circ} \mathrm{C}\right] \quad 248-249 \quad 248-249$

Schwefelsäure-Wasser-Reaktion $\quad 270 ; 296 ; \quad 270$;

$\lambda_{\max }[\mathrm{nm}] \quad 455 \quad 295 ; 456$

Kober-Reaktion $\lambda_{\max }[\mathrm{nm}] . \quad 519,5 \quad 519$

IR-Spektrum in $\mathrm{KBr} \quad$ identisch

Etwa 15 Jahre nach diesen ersten Untersuchungen zum Nachweis von Steroidepoxiden waren ins Land gegangen, als sich neue Möglichkeiten abzeichneten, mit Hilfe der kombinierten Gaschromatographie/Massenspektrometrie (GC/MS) kleinste Mengen von Steroidhormonen spézifisch nachzuweisen.

Grundsätzlich gibt es zwei Anwendungstechniken der kombinierten GC/MS, die man sich zum Nachweis sowie zur quantitativen Bestimmung nutzbar machen kann.

1. Beim üblichen Verfahren der MS wird die nachžuweisende Verbindung, die durch ein Substanzeinlaß-System in das Gerät gebracht wird, zunächst ionisiert und dabei teilweise in kleinere positiv geladene Bruchstücke gesspālten. Diese werden in einem elektrischen Feld beschleunigt und in einem magnetischen Feld abgelenkt. Durch kontinuierliche Veränderung der Magnetfeldṣtärke wer- 
den die gebildeten Ionen entsprechend ihrer Masse, d.h. ihrer $\mathrm{m} / \mathrm{e}$-Werte, auf dem Detektor abgebildet; dieses ist dann das Massenspektrum.

2. Bei der zweiten Technik werden Magnetfeldstärke und Beschleunigungsspannung auf einen substanzcharakteristischen $\mathrm{m} / \mathrm{e}$-Wert fest eingestellt, der entweder dem Molekülion oder einem spezifischen Fragmention der zu untersuchenden Substanz entspricht; diese Technik wird als Massenfragmentographie, im Englischen etwas zutreffender ,selected ion monitoring “ bezeichnet.

Bei diesem Vorgehen wird die Substanz in der Regel über eine gaschromatographische Trennsäule in das Massenspektrometer eingegeben. Wenn ein Teil der in der Ionenquelle gebildeten Ionen noch vor dem Magnetfeld abgeleitet und registriert wird, so erhält man - neben dem substanzspezifischen Massenfragmentogramm - ein Gaschromatogramm, das den Totalionenstrom wiedergibt (Abb. 1).

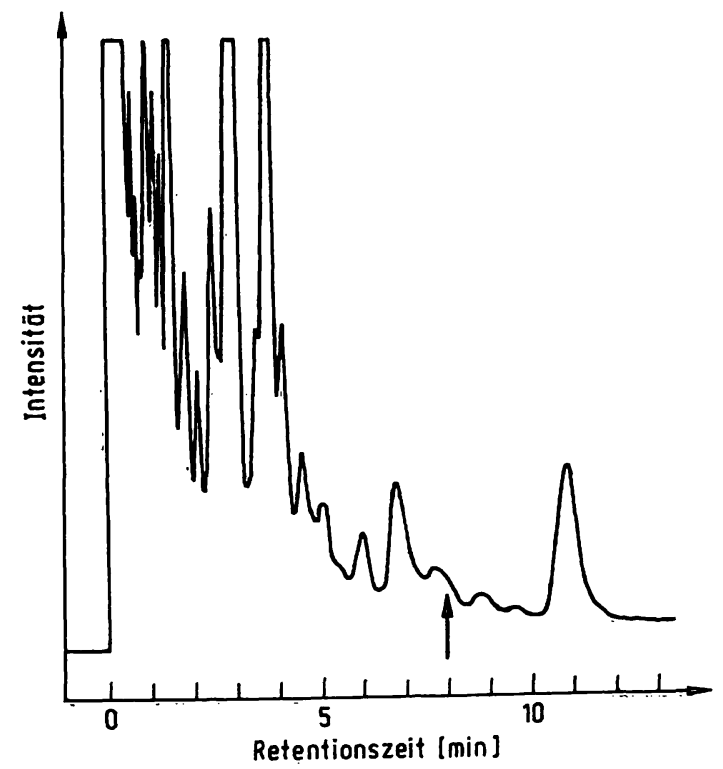

Abb. 1. Gaschromatogramm eines gereinigten und mit Essigsäureanhydrid behandelten Extraktes, der nach Inkubation von Östrätetraênol mit Rattenlebermikrosomen erhalten wurde. Als Detektor diente die Totalionenstromsonde des Massenspektrometers; der Pfeil zeigt die Retentionszeit an, bei der $16 \alpha, 17 \alpha$-Epoxyöstratrienolacetat zu erwarten ist.

Dieses substanz-unspezifische Signal entspricht etwa demjenigen eines Flammenionisationsdetektors. Bei einer
Retentionszeit von 7,9 min für authentisches $16 \alpha, 17 \alpha$ Epoxyöstratrienol (siehe Pfeil) erkennt man in diesem unspezifischen Chromatogramm keinen Peak, der von den übrigen Verunreinigungen aus dem biologischen Material abgetrennt ist. Im Gegensatz dazu wird bei der spezifischen Registrierung des m/e-Wertes 270 nur ein einziger Peak im Massenfragmentogramm erkennbar (Abb. 2), der dieselbe Retentionszeit wie die authentische Substanz aufweist. Das hier beschriebene Verfahren dient einerseits dem Nachweis, andererseits der quantitativen Bestimmung sehr kleiner Substanzmengen im biologischen Material.

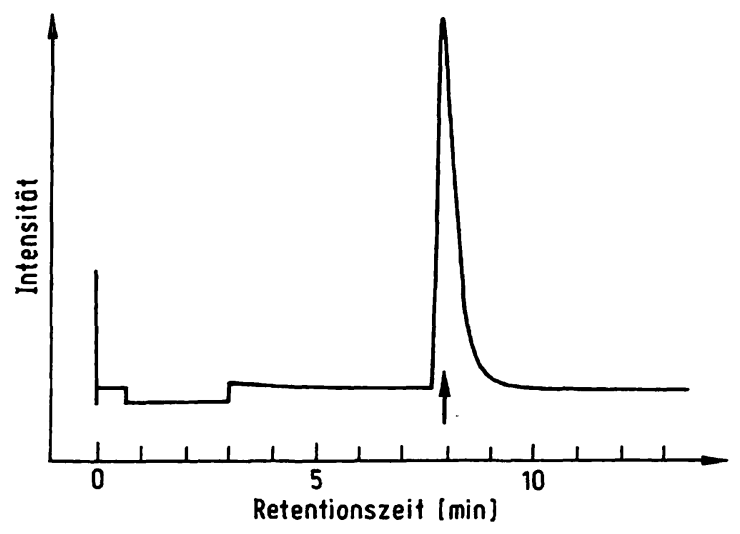

Abb. 2. Massenfragmentogramm eines gereinigten und mit Essigsäureanhydrid behandelten Extraktes, der nach Inkubation von Östratetraenol mit Rattenlebermikrosomen erhalten wurde. Für den substanzspezifischen Nachweis von $16 \alpha, 17 \alpha$-Epoxyöstratrienolacetat wurde das Massenspektrometer auf den m/e-Wert 270 eingestellt.

Der massenfragmentographische Nachweis, bei dem nur ein einzelnes Ion registriert wird, ist zwar besser als alle anderen chromatographischen Verfahren geeignet, bekannte Metaboliten in biologischem Material nachzuweisen und quantitativ zu bestimmen; die endgültige Identifizierung eines bisher nicht bekannten Metaboliten erfordert jedoch die Aufnahme eines kompletten Massenspektrums, wobei Molekülionen und zahlreiche Fragmentionen einer Substanz in charakteristischen Intensitätsverhältnissen registriert werden. Nachdem durch die kombinierte GC/MS sowie die Massenfragmentographie die analytischen Voraussetzungen für den spezifischen Nachweis kleinster Steroidmengen gegeben waren, konnte das Problem der Bildung von. Steroidepoxiden erneut aufgegriffen werden. 

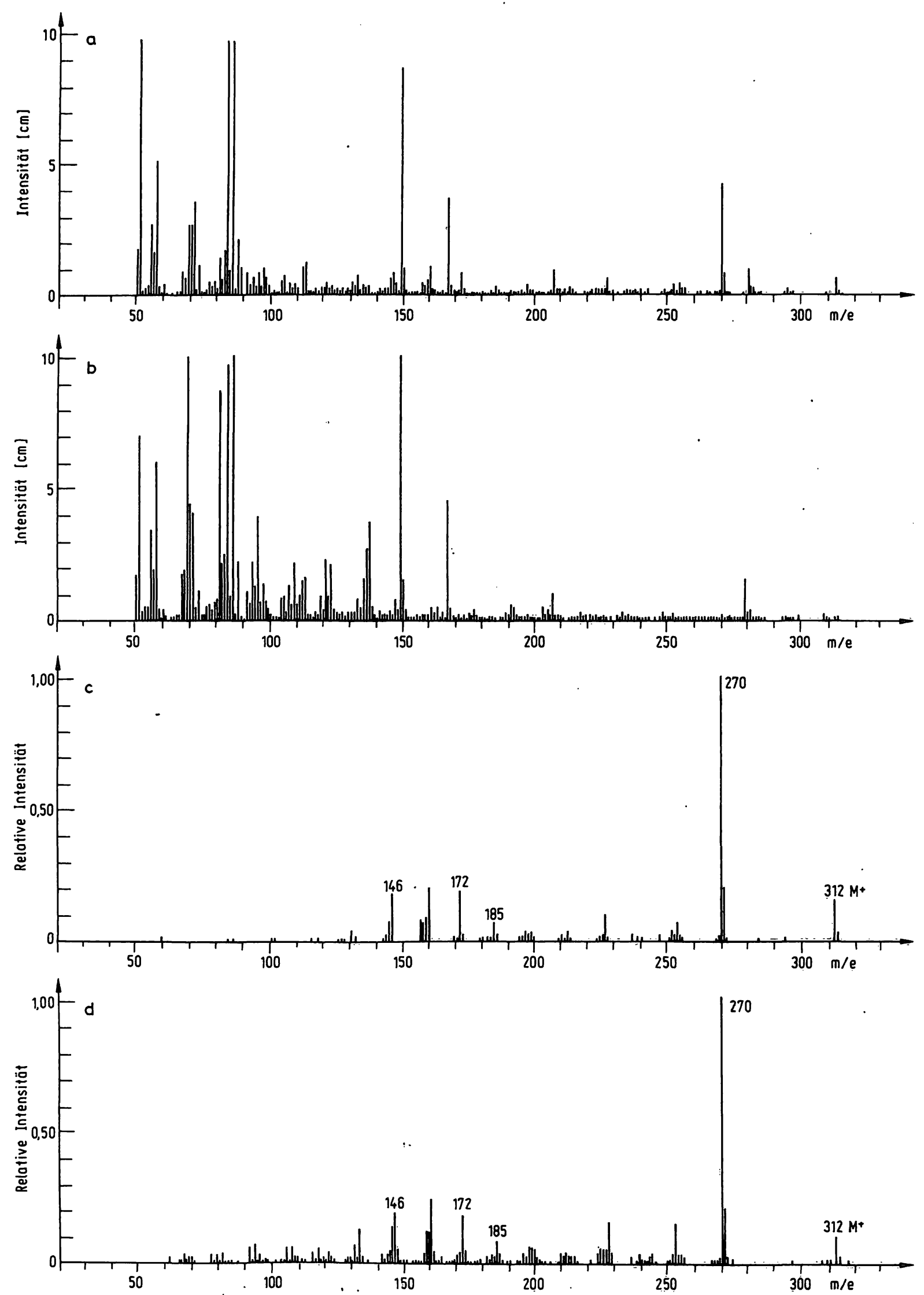

Abb. 3. Massenspektren.

a) Massenspektrum von $16 \alpha, 17 \alpha$-Epoxyöstratrienolacetat, aufgeżeichnet $470 \mathrm{~s}$ nach İnjektion eines gereinig̈ten und mit Essigsäureanhydrid behandelten Inkubationsextraktes.

b) Untergrundspektrum, aufgezeichnet $460 \mathrm{~s}$ nach der Injektion.

c) Massenspektrum von $16 \alpha, 17 \alpha$-Epoxyöstratrienolacetat wie unter (a) jedoch nach Subtraktion des Untergrundspektrums (b) und Normalisierung auf den Base-Peak (m/e 270).

d) Massenspektrum von authentiṣchem $16 \alpha, 17 \alpha$-Epoxyöstratrienolacetat. 
Zunächst befaßten wir uns mit der Bildung des Epoxids der Östrogenreihe $(16 \alpha, 17 \alpha$-Epoxyöstratrienol). $\mathrm{Zu}$ diesem Zwecke wurde tritiiertes $\Delta^{16}$-Östratrienol mit der Mikrosomenfraktion der Rattenleber in Gegenwart des NADPH-generierenden Systems unter Sauerstoff inkubiert; um eine rasche Hydrolyse des gebildeten Epoxids durch die mikrosomale Hydrolase zu verhindern, wurde Styroloxid zugesetzt, das die Epoxidhydrolase kompetitiv hemmt.

Die Inkubationsansätze wurden mit Ether/Chloroform extrahiert und die Acetylester-Derivate der Steroide gebildet.

Die dünnschichtchromatographische Auftrennung liefert drei Fraktionen, von denen die unpolare das Ausgangsprodukt ( $\Delta^{16}$-Östratrienol) enthält. Die Fraktion mit mittlerer Polarität entspricht der Wanderungsgeschwindigkeit von $16 \alpha, 17 \alpha$-Epoxyöstratrienol, während die: polare Fraktion im Bereich des $R_{F}$-Wertes für 16,17-Epiöstriol lag.

Ein Teil der Fraktion, die das vermutete Epoxid enthielt, wurde in eine Gaschromatographiesäule injiziert, die mit dem MS gekoppelt war. Bei der Retentionszeit von authentischem Epoxyöstratrienol wurde ein Massenspektrum des Metaboliten aufgezeichnet, wie es im oberen Teil der Abbildung 3 wiedergegeben ist. Etwa 10 s zuvor wurde ein „Untergrund-Spektrum" registriert, das als zweites Spektrum der Abbildung 3 zu sehen ist. Nach Subtraktion des Untergrundspektrums und nach Normierung auf den „base peak" $(=100)$ erhält man ein bereinigtes Massenspektrum des aus $\Delta^{16}$-Östratrienol entstandenen Metaboliten (Abb. 3, drittes Spektrum). Vergleicht man dieses bereinigte Massenspektrum mit dem- jenigen von authentischem Referenzmaterial (Abb. 3, unteres Spektrum) so besteht kein Zweifel, daß die hier isolierte Verbindung mit $16 \alpha, 17 \alpha$-Epoxyöstratrienol identisch ist (vgl. l.c. (9)). Damit kann die früher postulierte Bildung des Östrogenepoxids und damit auch der Steroidexpoxid-Stoffwechselweg als endgültig bewiesen angesehen werden (Abb. 4).

Wie bereits erwähnt, kommen nicht unerhebliche Mengen von $\Delta^{16}$-Verbindungen der $C_{19}$-Steroide, also der Androgene, beim Menschen vor (2-5). Diese $\Delta^{16}$.Verbindungen sind als stark riechende Sexuallockstoffe, d.h. Pheromone, bekannt geworden, die beim Eber eine wichtige Rolle spielen, allerdings nach neueren Befunden auch im Sellerie vorkommen sollen (10).

Uber die Biogenese von $\Delta^{16}-C_{19}$-Steroiden herrscht bis heute noch keine vollständige Klarheit, doch gibt es experimentelle Hinweise, daß sie aus Pregnenolon und Progesteron entstehen (11). Um festzustellen, ob auch die $\Delta^{16}$-Verbindungen der $C_{19}$-Steroide zur Epoxidbildung befähigt sind, wurde tritiiertes 4,16-Androstadien-3-on mit der Mikrosomen-Fraktion der Rattenleber unter den oben angegebenen Bedingungen inkubiert.

Die Aufarbeitung erfolgte ebenfalls analog dem früheren Vorgehen und ergab wiederum drei Fraktionen, von denen sich diejenige mit mittlerer Polarität wie ein 16,17-Epoxid von 4-Androsten-3-on verhielt. Die polare Fraktion zeigte das gleiche Verhalten wie die $16 \beta$, $17 \alpha$-Dihydroxyverbindung, ein Hydrolyseprodukt des Androgen-Epoxids.

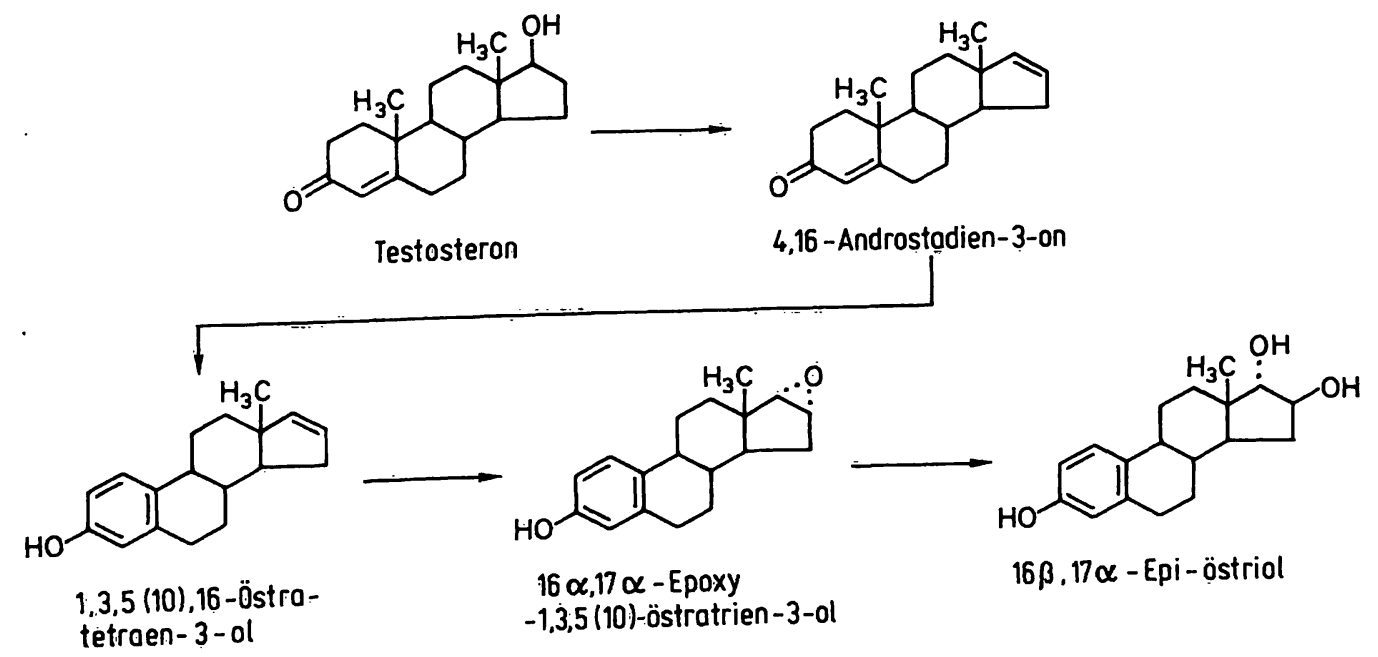

Abb. 4. Biogenese von $16 \alpha, 17 \alpha$-Epoxyöstratrienol.

J. Clin. Chem. Clin. Biochem. / Vol. 18, 1980 / No. 12 
Die kombinierte GC/MS der Epoxid-ähnlichen Fraktion ergab ein Massenspektrum, wie es im unteren Teil der Abbildung 5 dargestellt ist. Ein Vergleich mit dem Spektrum der authentischen Referenzverbindung
(Abb. 5, oberer Teil) legt den Schluß nahe, daß der hier isolierte Metabolit von 4,16-Androstadien-3-on mit dem $16 \alpha, 17 \alpha$-Epoxid von 4-Androsten-3-on identisch ist (vgl. l.c. (12)).
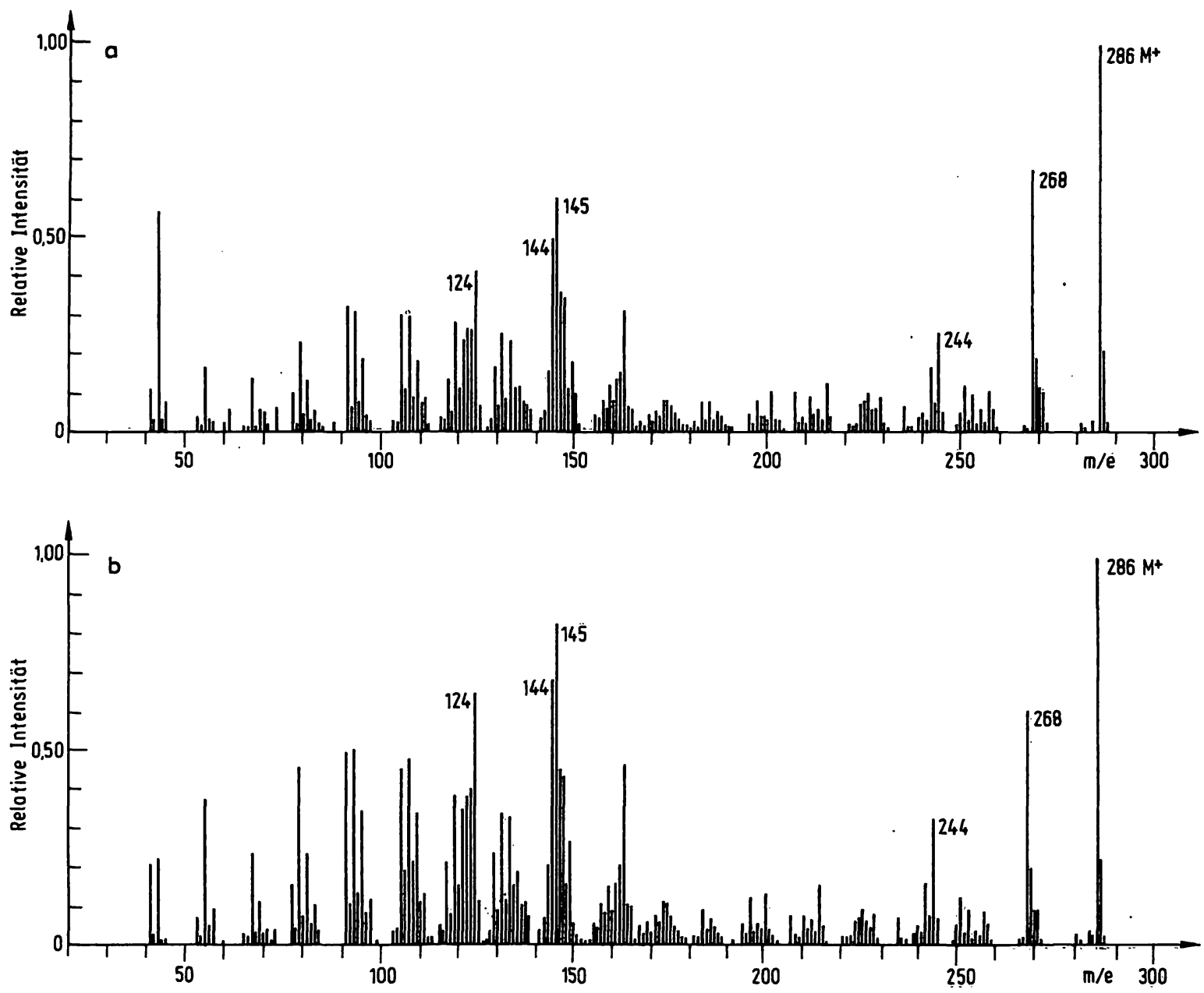

Abb. 5. Massenspektren.

a) Massenspektrum von authentischem $16 \alpha, 17 \alpha$-Epoxy-4-androsten-3-on.

b) Massenspektrum von $16 \alpha, 17 \alpha$-Epoxy-4-androsten-3-on, aufgenommen während der Gaschromatographie eines gereinigten Extraktes, der nach Inkubation von 4,16-Androstadienon mit Rattenlebermikromen erhalten wurde.<smiles>CC12CCC(=O)C=C1CCC1C2CCC2(C)C3C[C@H](O3)C12</smiles>

$\mathrm{H}_{5} \mathrm{IO}_{6}$

$16 \alpha .17 \alpha-$ Epoxy -4-androsten-3-on<smiles>CC12CCC(=O)C=C1CCC1C2CCC2(C)C1CC1OC12</smiles>
$\stackrel{\mathrm{H}_{5} \mathrm{IO}_{6}}{\longrightarrow}$ -4-ondrosten-3-on

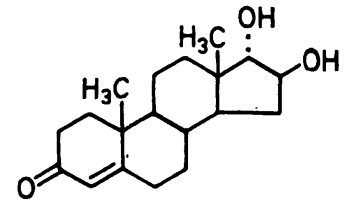

$16 \beta, 17 \alpha-$ Dihydroxy-4-ondrosten $-3-0 n$<smiles>CC12CCC(=O)C=C1CCC1C2CCC2(C)C1C[C@@H](O)C2O</smiles>

$16 \alpha, 17 \beta-$ Dihydroxy-4 - androsten -3 - on
Allerdings muß einschränkend gesagt werden, daß für das entsprechende $16 \beta, 17 \beta$-Epoxid ein gleichartiges Spektrum zu erwarten ist. Um in diesem Punkte Klarheit zu erlangen, wurde eine mikrochemische Reaktion mit Periodsäure durchgeführt (Abb. 6).

Abb. 6. Hydrolyse von $16 \alpha, 17 \alpha$ - und $16 \beta-17, \beta$-Epoxysteroiden mit Periodsäure. 
Wenn das $16 \alpha, 17 \alpha$-Epoxid mit Periodsäure umgesetzt wird, bildet sich ausschließlich das $16 \beta, 17 \alpha$-trans-Diol

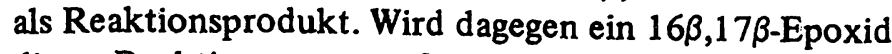
dieser Reaktion unterworfen, so entstehen als Reaktionsprodukte sowohl das $16 \beta, 17 \alpha$-transDiol als auch das $16 \alpha, 17 \beta$-trans-Diol.

Das während der Inkubation entstandene AndrogenEpoxid lieferte beim Umsatz mit Periodsäure ausschließlich die $16 \beta, 17 \alpha$-Dihydroxyverbindung. Daraus kann geschlossen werden, daß auch in der. Androgenreihe die $\Delta^{16}$-Verbindungen zu den entsprechenden $16 \alpha, 17 \alpha$ Epoxiden umgesetzt werden (vgl. l.c. (12)).

Auch in der Reihe der $\mathrm{C}_{21}$-Steroide sind $\Delta^{16}$.Verbindungen nicht unbekannt. So konnte $\Delta^{16}$-Progesteron beim Menschen nachgewiesen werden. Die Biogenese von 16-Dehydroprogesteron aus Progesteron verläuft vermutlich über $17 \alpha$-Hydroxyprogesteron $(13,14)$.

Die chemische Synthese von tritiiertem 16-Dehydroprogesteron aus tritiiertem Pregnenolon verläuft über acht Stufen, wobei zunächst eine Bromierung und dann eine
Abspaltung von $\mathrm{HBr}$ von den C-Atomen 16 und 17 erfolgt. Durch Umwandlung der $\Delta^{5}$-3 $\beta$-Hydroxyverbindung in eine $\Delta^{4}$-3-Oxokonfiguration erhält man in $15 \%$ Ausbeute das gewünschte 16-Dehydroprogesteron. Die Inkubation dieser Verbindung und die Auftrennung der dabei gewonnenen Fraktionen erfolgten in gleicher Weise wie für die $\mathrm{C}_{18}$ - und $\mathrm{C}_{19}$-Steroide beschrieben (15).

Nach 10 min Inkubation von 16-Dehydroprogesteron konnte kein Epoxid nachgewiesen werden; allerdings entstanden in erheblichem Umfang Ring-A-reduzierte $\Delta^{16}$. Verbindungen, nämlich das $5 \alpha$-reduzierte Dion und die $5 \alpha$-reduzierte $3 \beta$-Hydroxyverbindung (Abb. 7). Außerdem wurde eine 6-Hydroxyverbindung gebildet. Nach 120 min Inkubation wurden zwei Metaboliten von 16-Dehydroprogesteron gefunden, bei denen es sich um Epoxide handelte.

Das 16 $\alpha, 17$-Epoxid von Progesteron war nur in sehr geringen Mengen nachweisbar; es ist nicht auszuschließen, $\mathrm{da}$ es sich hierbei lediglich um ein Autoxidationsprodukt handelt, das als Artefakt bei der analytischen Aufarbeitung entsteht. Im Gegensatz dazu entstand das $16 \alpha, 17$ -<smiles>CC(C)(C)C1=CC[C@H]2C3CCC4CC(=O)CCC4(C)C3CCC12C</smiles>

$5 \alpha$-Pregn-16-en-3,20-dion<smiles>CC(C)(C)C1=CC[C@H]2C3CCC4CC(O)CCC4(C)C3CCC12C</smiles>

$3 \beta-$ Hydroxy $-5 \alpha-p r e g n$ $-16-$ en-20-on<smiles>CC1(C)CC2C(CC3C4CCC5CC(O)CCC5(C)C4CCC23C)O1</smiles>

$16 \alpha, 17$ - Epoxy - $3 \beta$ - hydroxy

$-5 \alpha$-pregnan -20 -on

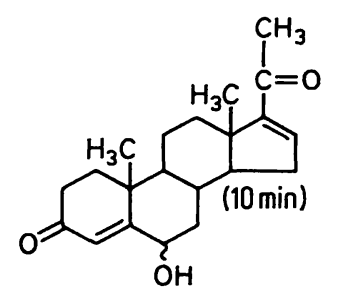

6-Hydroxy - 16 - dehydroprogesteron
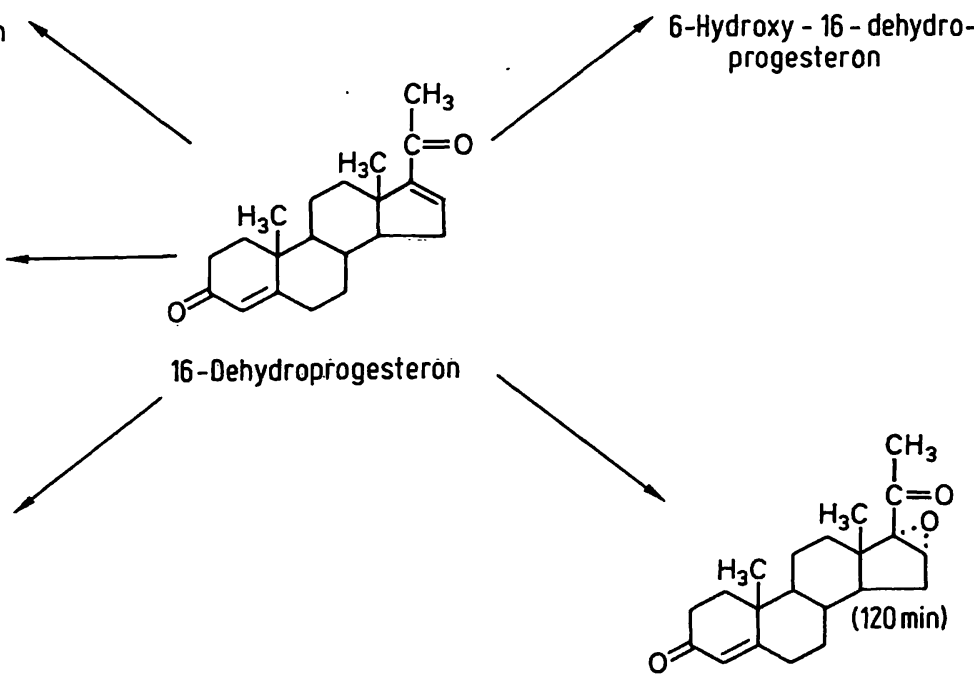

$16 \alpha, 17$ - Epoxyprogesteron

Abb. 7. Stoffwechselprodukte von 16-Dehydroprogesteron nach Inkubation mit Rattenlebermikrosomen. 
Epoxid der Ring-A-reduzierten Verbindung in größerer Menge und konnte massenspektrometrisch durch Ver- gleich mit der authentischen Verbindung eindeutig identifiziert werden (Abb. 8) (15).
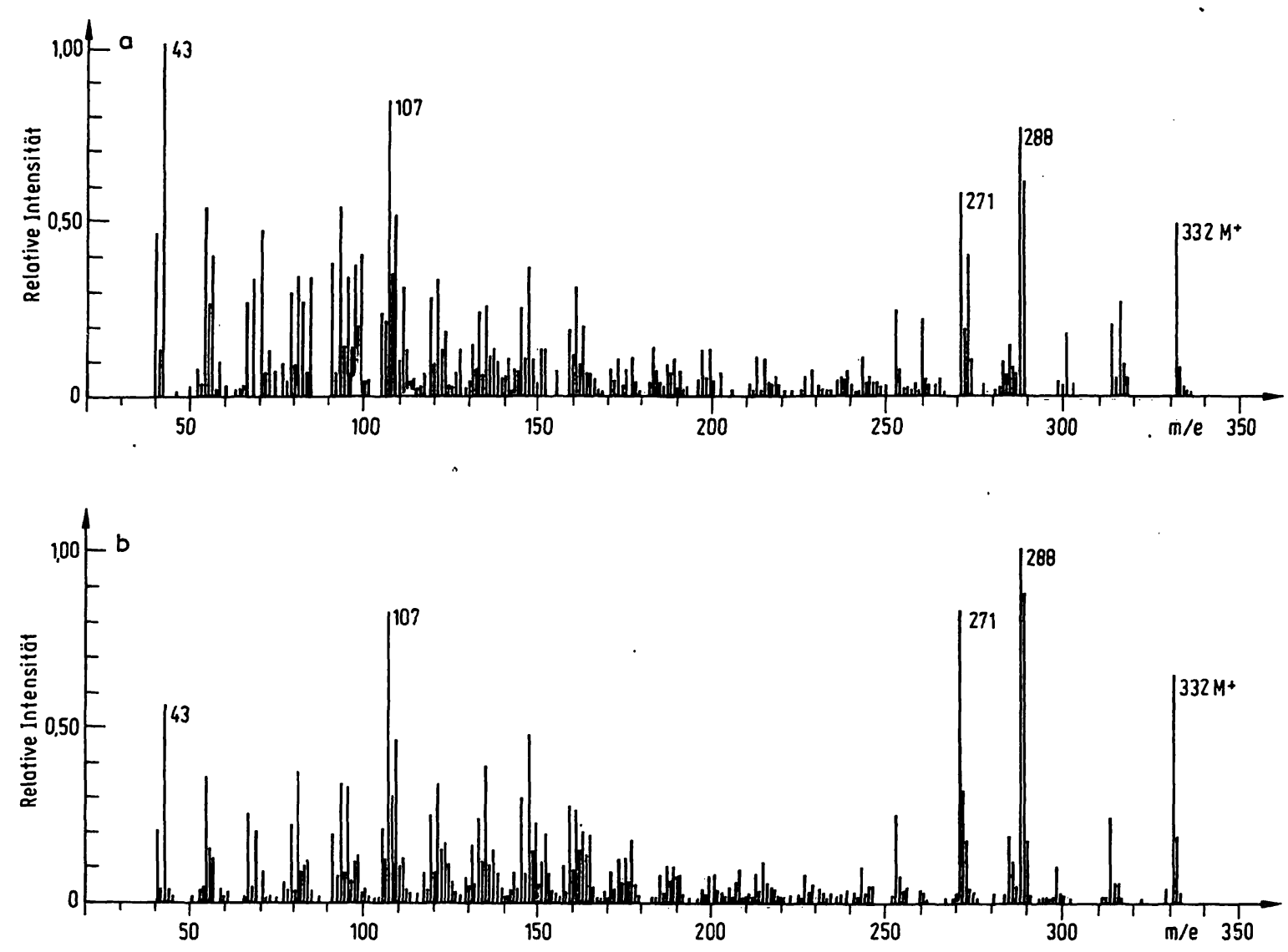

Abb. 8. Massenspektren.

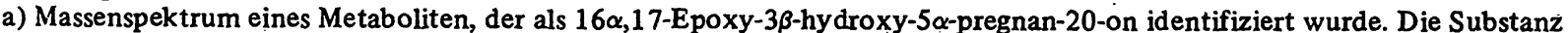
wurde nach Inkubation von 16-Dehydroprogesteron mit Rattenlebermikrosomen isoliert.

b) Massenspektrum von authentischem 16 $\alpha, 17$-Epoxy-3 $\beta$-hydroxy-5 $\alpha$-pregnan-20-on.

Diese Befunde legen den Schluß nahe, daß die Epoxide von $\mathrm{C}_{21}$-Steroiden langsamer gebildet und auch langsamer wieder umgesetzt werden. Wir untersuchten deshalb den Stoffwechsel von 16 $\alpha$,17-Epoxyprogesteron und stellten fest, daß 16ß-Hydroxyprogesteron als Hauptmetabolit entstand (Abb. 9). Die Bildung dieses Reaktionsproduktes kann über eine 16-Oxoverbindung als Intermediat erfolgen.

Faßt man die hier vorgetragenen Ergebnisse zusammen, so ergibt sich folgendes Bild (Abb. 10): Die $\Delta^{16}$-Verbindungen von $\mathrm{C}_{18^{-}}, \mathrm{C}_{19^{-}}$- und $\mathrm{C}_{21}$-Steroiden werden im Stoffwechsel zu 16 $\alpha, 17 \alpha$-Epoxiden umgesetzt. Die
Epoxidierung erfolgt bei den Östrogenen und Androgenen rasch, bei Progesteron deutlich langsamer. Die Epoxide der $\mathrm{C}_{18}$ - und $\mathrm{C}_{19}$-Steroide sind nur kurzlebige Zwischenprodukte, die durch die mikrosomale Hydrolase rasch zu den trans-Diolen aufgespalten werden. Die Eigenschaften und die mögliche biologische Bedeutung der $\mathrm{C}_{18}$-Steroidepoxid-Hydrolase wurden von Oesch und Mitarbeitern ausführlich untersucht (16). Das entsprechende Epoxid von Progesteron hat wohl auf Grund des sterischen Einflusses der Seitenkette eine deutlich längere Lebensdauer und wird nicht zu einem trans-Diol, sondern zu einer Monohydroxy-Verbindung umgewandelt (vgl. Abb. 9).

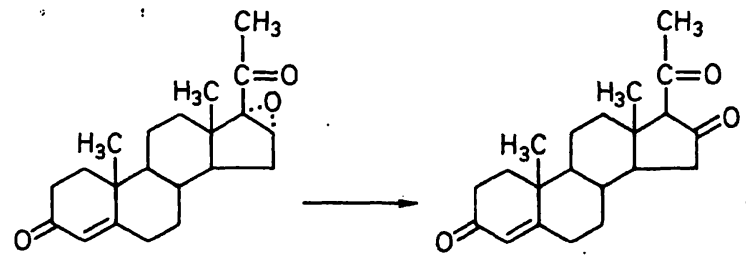

$16 \alpha, 17$ - Epoxyprogesteron
16 -Oxoprogesteron

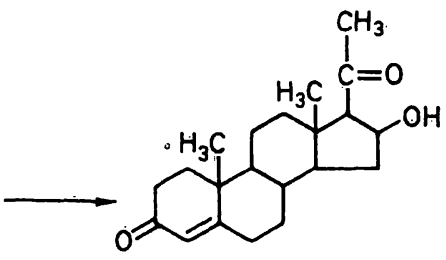

16. $\beta$-Hydroxyprogesteron
Abb. 9. Stoff wechsel von $16 \alpha$, 17-Epoxyprogesteron in Rattenlebermikrosomen. 


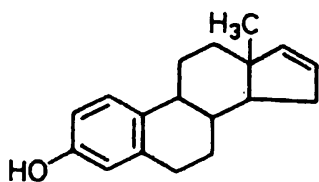

$1,3,5(10), 16-$ östrotetraen $-3-$ ol

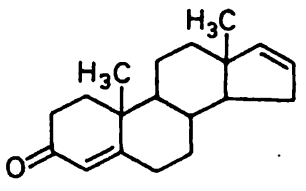

4.16-Androstadien-3-on

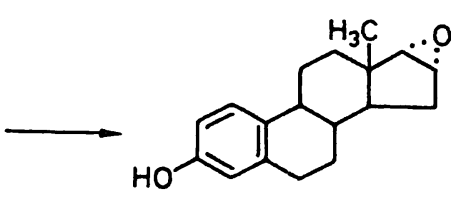

$16 \alpha, 17 \alpha-$ Epoxy$-1,3,5(10)$-östratrien $-3-0$

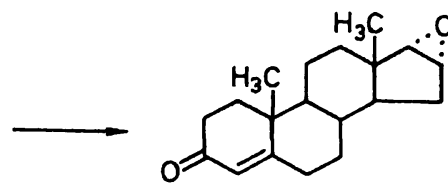

$16 \alpha, 17 \alpha-$ Epoxy -4 -androsten $-3-$ on

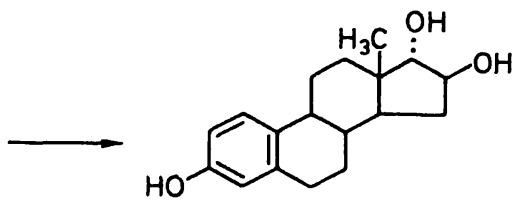

$16 \beta .17 \alpha-$ Epi-östriol

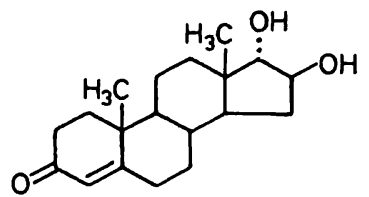

$16 \beta, 17 \alpha$ - Dihydroxy-4 -ondrosten -3 - on

Abb. 10. Biogenese und Stoffwechsel der Epoxide von $C_{18}$ - und $C_{19}$-Steroiden.

Die Tatsache, daß im Stoffwechsel von Östrogenen, Androgenen und Gestagenen Epoxide als normale Reaktionsprodukte entstehen, führt zu der Frage ihrer biologischen Bedeutung. Zur Zeit können zu diesem Punkte nur Spekulationen geäußert werden. Vom Standpunkt des Steroidstoffwechsels liegt der Gedanke nahe, daß eine Epoxidierung und anschließende Hydrolyse zu Reaktionsprodukten führt, die im Vergleich zu den Ausgangsverbindungen eine deutlich höhere Polarität aufweisen und dadurch - sei es durch Konjugierung oder auf Grund der erhöhten Wasserlöslichkeit - leichter ausgeschieden werden können. Zieht man einen Vergleich mit den Epoxiden der polycyclischen aromatischen Kohlenwasserstoffe, so ergibt sich die Frage, ob die hier nachgewiesenen Steroidepoxide eine ähnliche carcinogene Wirkung besitzen, wie sie bei den Kohlenwasserstoffen nachgewiesen worden ist. Erste Untersuchungen lassen vermuten, daß Steroidepoxide - so wie auch die Epoxide der Kohlenwasserstoffe - an Proteine und Peptide gebunden werden; dieses geschieht unter Aufspaltung des Epoxid-Rings. Es ist nicht un- realistisch, anzunehmen, daß Steroidepoxide auch mit Desoxyribonucleinsäuren reagieren und dadurch genetische Veränderungen herbeiführen könnten.

Was die mögliche Carcinogenität der Steroidepoxide anbetrifft, so haben frühere Untersuchungen in den sechziger Jahren gezeigt, daß die Verabreichung von $16 \alpha$, $17 \alpha$-Epoxyöstratrienol an Ratten die Inzidenzrate von Carcinomen nicht erhöhte (17). Diese vorläufige Studie bedarf zweifellos der Nachprüfung durch moderne Methoden der Zellbiologie. Erst wenn hier eindeutige Ergebnisse vorliegen, kann die seit Jahren immer wieder diskutierte Frage einer Carcinogese durch Steroidhormone beantwortet werden.

\section{Dankvermerk}

Herm Priv. Doz. Dr. Lothar Siekmann bin ich für die großzügige Überlassung experimenteller Daten, für anregende Diskussionen und für seine Mitwirkung bei der Abfassung des Manuskripts zu großem Dank verpflichtet. Ein Teil der Untersuchungen wurde von Herrn Dr. B. Disse und Frau Sibylle Martin durchgeführt.

\section{Literạtur}

1. Brophy, P. J. \& Gower, D. B. (1973), Biochem. Soc. Trans. $1,181-184$.

2. Brooksbank, B. W. L., Cunningham, A. E. \& Wilson, D. A. A. (1969), Steroids 13, 29-50.

3. Brooksbank, B. W. L., Wilson, D. A. A. \& Gustafsson, J. A. (1972), Steroids Lipids Res. 3, 263-285.

4. Brooksbarik, B. W. L., Wilson, D. A. A. \& MacSweeney, D. A. (1972), J. Endocrinol. 52, 239-251.

5. Brooksbank, B. W. L. (1962), J. Endocrinol. 24, 435-444.

6. Knuppen, R. \& Breuer, H. (1962), Acta Endocrinol. 42, 129-134.

7. Breuer, H. \& Knuppen, R. (1961), Biochim. Biophys. Acta $49,620-621$.

8. Knuppen, R. \& Breuer, H. (1962), Hoppe-Seylers Z. Physiol. Chem. 328, 226-234.

9. Siekmann, L., Thull, P. \& Breuer, H. (1980), Acta Endocrinol. 95, 49-57.

10. Claus, R. \& Hoppen, H. (1979), Experientia 35, 1674.

11. Brophy, P. J. \& Gower, D. B. (1972), Biochem. J. 128, 945-952.

12. Disse, B., Siekmann, L. \& Breuer, H. (1980), Acta Endocrinol. 95, 58-66.

13. Ball, J. H. \& Kadis, B. (1964), Steroids 4, 533-538.

14. Armstrong, A. A. \& Kadis, B. (1970), Steroids 15, 737749.

15. Siekmann, L., Disse, B. \& Breuer, H. (1980), J. Steroid Biochem. 13, 1181-1205.

16. Bindel, U., Sparrow, A., Schmassmann, H., Golan, M., Bentley, P. \& Oesch, F. (1979), Eur. J. Biochem. 97, 275281.

17. Breuer, H., Knuppen, R. \& Schmähl, D. (1965), Z. Krebsforsch. $66,549-551$.

Prof. Dr. H. Breuer Institut für Klin. Biochemie Venusberg D-5300 Bonn 1 
Hansjürgen Staudinger dankte der Deutschen Gesellschaft für Klinische Chemie für die Ehrung, Mitwirkenden und Gästen für die festliche Gestaltung des Nachmittags:

„Ja oder Nein ist die Frage; ist es besser, sich zu freuen oder sein Gesicht zu verhüllen? Ich bin entschlossen, mich zu freuen. Betreiben wir Wissenschaft wegen der Preise oder der Ehre oder zu unserer Freude? Ich hoffe, daß ich Wissenschaft immer zu meiner Freude betrieben habe. Ein Preis kann für einen 66jährigen kein Ansporn mehr sein, sondern belohnt die zurücklegende Arbeit durch ,erneute Freude".

Herr Staudinger dankte den Freiburger Barock-Solisten für ihren musikalischen Beitrag zur festlichen Gestaltung der Feierstunde. Er dankte Herm Kickhöfen dafür, daß die Feierstunde im Max-Planck-Institüt stattfinden durfte, für seine einführende Begrüßung und sein Wortspiel zum Medaillen-Patron Scherer und Scherer, einem Künstler: Einer der denkt, einer der handelt, spreche ihn an und ehre ihn. Herm Kruse-Jarres dankt Staudinger für die Mühen der Organisation. Durch die Worte von Frau Kersten fühle er sich richtig beurteilt, da der Irrtum als zur Wissenschaft gehörend liebenswürdig erwähnt wurde.

Für ihn zähle nicht allein der Erfolg, sondern vor allem die Liebe, mit der Wissenschaft betrieben werde. Der Dank seiner Schüler an ihren Lehrer erinnere ihn an seinen hier anwesenden Lehrer Franz Büchner, der ihn als Schüler angeregt habe, Untersuchungen über den Zusammenhang des Cholesterins der Nebenniere mit den Sterioden der Nebenniere anzustellen.

Staudinger erinnert an Mannheim und Herrn Kihm als Verwaltungsdirektor, dessen weitblickende sachliche Unterstützung er mit Dank vermerkt, und ging dann auf die Ausführungen von Herrn Kattermann zur Entwicklung der Klinischen Chemie in Mannheim, die ihn ja teilweise selbst betrafen, ein: Die zunehmenden Analysenzahlen machten ihn nachdenklich. Fortschritte der Wissenschaft seien immer auch methodisch apparativer Fortschritt gewesen. Früher seien Wissenschaft und Routine Handarbeit gewesen, die Ausführenden Handwerker; heute würden industriell im Takt Resultate geschafft. Eine moralische Wertung dieser Entwicklung sei nicht möglich.

Für den Festvortrag von Herrn Breuer und die gegenseitigen Beziehungen dankt Staudinger herzlich. Neben vielen guten Kontakten habe es auch echten wissenschaftlichen Wettstreit gegeben. Dabei sei Breuer nach seiner Ansicht immer ein ebenbürtiger Mitstreiter gewesen: solcher Wettstreit sei der Wissenschaft förderlich, die Verdienste der Wissenschaftler bilden ein Synzytium. Auch bestätige der Vortrag von Breuer die parawissenschaftliche Aussage, daß Forschung am ersten vermeintlichen Ziel meist nur Aufenthalt mache und auf den methodischen Fortschritt warte, um dann zu weiteren Erkenntnissen zu führen.

Herr Staudinger schloß seine Ausführungen mit einem wiederholten herzlichen Dank an alle Gestalter und Gäste.

F. Körber, Berlin 